\title{
Lúdica como factor potenciador de la creatividad en los niños de Educación Preescolar
}

\section{Playful as a creator of creativity in Preschool Children}

\author{
Ana Esther Oñate-Gonzalez \\ anesogo@hotmail.com \\ Institución Educativa Elba Solano, Barrancas. \\ Colombia \\ https://orcid.org/0000-0002-3372-6903
}

Recibido: 17 de noviembre de 2019

Aprobado: 20 de diciembre de 2019

\begin{abstract}
RESUMEN
El estudio que se desarrollará tiene como propósito principal describir la Lúdica como potenciador de la Creatividad en los Niños de Educación Preescolar. Es una investigación de naturaleza cualitativa, adscrita al paradigma interpretativo, a fin de comprender el sentido de los significados que le atribuyen los actores sociales al fenómeno, coincidiendo con la dimensión ontológica de la misma, tomando en cuenta su realidad holística, integradora. En cuanto a los resultados se tiene como principio fundamental que la escuela debe ser una institución que aporte a la formación integral de las niñas y niños, acompañando el desarrollo de personalidades para continuar su hacer en el mundo en armonía con los mejores ideales humanos.
\end{abstract}

Descriptores: Lúdica; potenciador; creatividad; niños; educación preescolar.

\begin{abstract}
The study that will be carried out has the main purpose of describing Playfulness as an enhancer of Creativity in Preschool Children of the Educational Institution Elba Solano Barrancas La Guajira Colombia. It is a qualitative research, attached to the interpretive paradigm, in order to understand the meaning of the meanings attributed by the social actors to the phenomenon, coinciding with its ontological dimension, taking into account its holistic, integrative reality oriented by a phenomenological methodology. Regarding the results, the fundamental principle is that the school must be an institution that contributes to the integral formation of girls and boys, accompanying the development of personalities to continue their work in the world in harmony with the best human ideals.
\end{abstract}




\section{Ana Esther Oñate-Gonzalez}

Descriptors: Playful; enhancer; creativity; children; preschool education.

\section{INTRODUCCIÓN}

Debido a los continuos avances de la ciencia y la tecnología, el mundo nos exige cada día más seres innovadores, capaces de solucionar problemas y resolver conflictos usando lo que el entorno le puede brindar. Así mismo se busca una estructura más compleja del pensamiento que permita hacer uso de las estrategias, diseños y enfoques que respondan a las necesidades de la sociedad. En diferentes países del mundo la falta de creatividad por parte de los estudiantes ha sido una problemática que ha subsistido por largos años y que aún es tema de controversias de algunos autores.

En el mismo sentido, en declaraciones realizadas por la UNESCO (2010), se manifiesta la necesidad de realizar reestructuraciones curriculares en los contenidos y métodos de enseñanzas utilizados con el objetivo de desarrollar habilidades de pensamiento creativo; dichas habilidades referidas a la originalidad, fluidez de ideas, innovación, factibilidad y viabilidad. En este marco de referencia, actualmente en Colombia se viene desarrollando una serie de documentos que apuntan a los cambios que se han manifestado hoy día en la educación. Sin embargo, son pocas las instituciones educativas del país que llevan a cabo procesos lúdicos para desarrollar este tipo de pensamiento que conlleve a un proceso de aprendizaje a feliz término.

Es así, como la educación está llamada a dotar sus instituciones educativas con personal docente comprometido con la disposición de enseñar y manejar situaciones de aprendizaje que ameriten actividades lúdicas creativas, por cuanto ella, es también un clamor de amor a la infancia encerrando lo que Delors (1997), manifiesta en el informe de la Comisión Interamericana sobre educación para el siglo XXI presentado a la UNESCO, "el niño es el futuro del hombre", lo que indica la relevancia de la formación bajo la concepción de la lúdica por ser un proceso inherente al desarrollo humano en toda su dimensión, dirigida a la búsqueda del sentido de lo aprendido.

De allí, la lúdica como experiencia cultural, es una dimensión transversal que atraviesa toda la vida, no son prácticas, actividades, ciencia, disciplina, ni mucho menos una 


\section{Ana Esther Oñate-Gonzalez}

nueva moda, sino un proceso inherente al desarrollo humano en toda su dimensional psíquica, social, cultural y biológica, ligada a la cotidianeidad, en especial a la búsqueda del conocer el por qué el aprendizaje no debe ser aburrido, sino entusiasta, agradable, para que el aprendizaje sea significativo y potenciador de las capacidades de los discentes.

\section{DESARROLLO TEÓRICO}

\section{La Educación Preescolar}

La educación preescolar cobra creciente relevancia y reconocimiento social porque interviene en los primeros años de vida de los niños, período en el que desarrollan su identidad personal, adquieren capacidades fundamentales de diversos órdenes y aprenden las pautas básicas para integrarse a la vida social. En el Jardín de niños, según la riqueza de las experiencias en las que participen, adquieren seguridad y confianza en sí mismos, fortalecen su curiosidad, sus capacidades de observar, prestar atención, formular preguntas y explicaciones; asimismo, aprenden a reconocer sus capacidades propias, a procesar información, y desarrollan su imaginación y creatividad. (SEP, 2008).

Hace 25 años, la visión de la "educación preescolar" era mucho más clara y estrecha que la actual. En esa época, el término se refería esencialmente a programas formales que se llevaban a cabo en ambientes de tipo escolar fuera del hogar, orientados a niños que estaban a punto de ingresar a la educación primaria. La educación preescolar evocaba una imagen de 25 niños de unos 4 a 5 años, sentados alrededor de pequeñas mesas en las que se dedicaban a dibujar o a hacer calzar triángulos de colores en un rompecabezas, bajo la supervisión de un docente profesional.

Los programas de educación o promoción del aprendizaje en niños menores de cuatro años probablemente no eran tomados en consideración o simplemente eran tratados como actividades de "guardería", motivo por el cual eran abordados por separado. Los esfuerzos no formales, organizados, comunitarios orientados a proporcionar atención precoz al desarrollo mental y social de los niños pequeños no eran comunes y no se incluían en las estadísticas preescolares.

Sin embargo, los tiempos están cambiando; se está desarrollando una visión más 


\section{Ana Esther Oñate-Gonzalez}

amplia de la educación preescolar, que abarca la educación o el aprendizaje durante todo el período que va desde el nacimiento hasta el ingreso a la escuela, en una amplia gama de ambientes formales y no formales. En consecuencia, los niños inscritos en "programas preescolares" pueden tener desde algunas semanas de edad hasta siete años y, en la actualidad, estos programas pueden ser:

Programas preescolares convencionales, administrados o autorizados por el gobierno. Programas no formales, que a veces son administrados por el gobierno pero que también son manejados por organizaciones no gubernamentales o por las comunidades; Programas de atención infantil y desarrollo integral que incluyen una componente educacional o de desarrollo psicosocial. (En algunos casos, estos programas operan fuera del sector educacional como parte, por ejemplo, de un sistema de seguridad social, pero reciben apoyo técnico de la educación. La organización responsable puede ser una agencia de gobierno, una ONG o una organización comunitaria).

Programas de capacitación de padres o adultos destinados a capacitarlos para que mejoren sus habilidades como "primeros profesores" de sus niños, en los cuales la educación de los niños se produce en los hogares. Estos cambios y la apertura de la definición están dándole dinamismo a la tarea de la educación preescolar pero también complican la actividad de organizar y poner en práctica los programas dificultando, a la vez, la descripción del "estado del arte" sobre la educación inicial y preescolar.

\section{Lúdica como Estrategia Pedagógica}

El abordaje de la lúdica se realiza desde la concepción de las nuevas tendencias educativas las cuales llevan el propósito de apoyar la formación integral entre los niños dentro o fuera de la escuela y comprometer tanto a la escuela como a las familias a ampliar sus valores para vincular las metas educativas con el ejercicio vivencial de niños, niñas y adolescentes.

Por su parte Mendoza, (2015, p. 308) reseñan en su artículo que la actividad lúdica como estrategia pedagógica es "fundamental en la educación ya que facilita la expresión, la espontaneidad, la socialización y el aprendizaje significativo, propiciando 


\section{Ana Esther Oñate-Gonzalez}

un ambiente satisfactorio, formación de una personalidad adulta, crítica, reflexiva, consciente de la realidad, capaz de promover y buscar alternativas de solución". Pues, fortalece los valores, con ello, estimula el conocimiento, de la misma forma, el pensamiento reflexivo, ya que coinciden en el carácter de la moralidad del individuo. Por lo tanto, debe formarse, en su quehacer en el medio social en el cual se desenvuelve y está inmerso.

La actividad lúdica, permite un desarrollo integral en el alumno, satisfacerse dentro de su entorno natural, conlleva a un aprendizaje espontáneo que, a través de esto, se aprenden las normas y pautas de comportamiento, se resaltan los valores y actitudes, se despierta la curiosidad, es decir, es necesidad en la persona cuando se comparte y se juega, se vive experiencias y se aprende las vivencias que lo preparan para confrontar responsabilidades en la sociedad de la que formará parte y se beneficia la comunicación y la creación, por ser una manera de expresión franca y motivadora.

En este sentido, el empleo docente de la lúdica pareciera que despliega novedades, pero a condición de que sobre salgan las orientaciones como los medios, así como se introduzcan el uso en una pedagogía distinta a la tradicional de transmisión pasiva y/o la destreza técnica; además, se consideren las desigualdades social y nacional, se recuerden los perfiles de aprendizaje del estudiante.

En este particular, se asocian los comentarios de Hidalgo y Valverde (2009, p. 11) donde señalan que la lúdica "permite fundamentar muchas de las decisiones sobre las estrategias de protección y promoción del desarrollo del alumno, es decir, siendo la actividad del juego, como método primordial en la implementación de estrategias, se integran los elementos metodológicos", pues, concibe actividad placentera para quienes participan y como medio para la realización de los objetivos programados en las diferentes actividades que se deseen proyectar o disponer. Es por ello, que la lúdica, fomenta en enriquecer el desarrollo de la personalidad, evidencia valores, puede orientarse a la adquisición de saberes, enclaustrando una amplia gama de actividades donde interactúan, la satisfacción, la creatividad, el conocimiento instantáneo y perenne.

No obstante, la lúdica debe ser tomada en cuenta especialmente en las aulas, pues, es rica en ambientes facilitadores de experiencias vivenciales. Desde esta 


\section{Ana Esther Oñate-Gonzalez}

perspectiva, el gran reto de la incorporación de la lúdica al ámbito escolar es encontrar buenas herramientas para difundir mejor lo que saben o lo que desean que aprendan los estudiantes, encontrar los mejores medios para hacer llegar a distintos destinatarios dentro de las aulas de clase, que les permita aprender con alegría, en medio de la cooperación y con disposición hacia el compartir.

Por tal razón, en el ámbito de la educación la lúdica aparece como herramientas con una prometedora capacidad de cambio en términos de los niveles educativos y de la igualdad de las oportunidades porque generan cambios efectivos, estrategias didácticas de los docentes y aprendizaje independiente y permanente según las necesidades de los individuos.

Por otra parte, el concepto de lúdica según Jiménez (2002) es tan amplio como complejo, pues se refiere a la necesidad del ser humano, de comunicarse, de sentir, expresarse y producir en los seres humanos una serie de emociones orientadas hacia el entretenimiento, la diversión, el esparcimiento, que nos llevan a gozar, reír, gritar e inclusive llorar en una verdadera fuente generadora de emociones. De allí que la lúdica fomenta el desarrollo psico-social, la conformación de la personalidad, evidencia valores, puede orientarse a la adquisición de saberes, encerrando una amplia gama de actividades donde interactúan el placer, el gozo, la creatividad y el conocimiento. Es así que la lúdica debería ser tomada en cuenta principalmente en los espacios escolares pues es rica en ambientes facilitadores de experiencias que mediante juegos, es necesario explicar cuanto más experiencias positivas y cuantas más realidades los niños conozcan, serán mucho más amplios y variados los argumentos de sus actividades, con respecto a la lúdica, es una dimensión del desarrollo humano que fomenta el desarrollo psicosocial, la adquisición de saberes, la conformación de la personalidad, es decir encierra una gama de actividades donde se cruza el placer, el goce, la actividad creativa y el conocimiento para tener más claridad ante la lúdica. En el mismo orden de ideas, los tipos de estrategias lúdicas se entienden como todas aquellas competencias que utiliza el ser humano, para los procesos de iniciación de las actividades. De acuerdo con, Campos (2010), según lo citado por Andrade, Carruyo y Hernández (2012), señala que las estrategias son las impuestas por el aprendizaje, inducidas por las estrategias cognitivas y que se involucran en el proceso 


\section{Ana Esther Oñate-Gonzalez}

de la información a partir del texto, que realiza un lector, aun cuando en el primer caso en énfasis se hace en materias y en el segundo en el aprendiz. Dentro de las técnicas y actividades a utilizar en las denominadas estrategias lúdicas como el juego educativo, libre, de análisis y lógicos.

Por otra parte, Danserau (2009) manifiesta que los tipos de estrategias son las que operan directamente sobre el material informativo y que se vinculan directamente los procesos de comprensión y apoyo como las que tratan de mantener el clima cognitivo al igual que necesario haciendo énfasis a la planificación, elaboración y programación de las metas u objetivos previsto alcanzar.

De igual manera, Beltrán (2007) expresan que los tipos de estrategias que tienen en cuenta para los diversos criterios de su naturaleza cognitivas, de apoyo y meta cognitiva dependiendo la función y la sensibilización, recuperación, evaluación las cuales se cruzan en la obtención de una clasificación que las divide en cuatro grupos de apoyo, como los procedimientos de personalización de conocimientos y metacognitivas. Es por ello que en las instituciones educativas de Colombia, los docentes deben emplear los tipos de estrategias puesto que las mismas contribuyen al desarrollo del aprendizaje de los alumnos, al aplicarlas por medio de algunas herramientas entre ellos los juegos educativos, los juegos libres, de análisis lógico, entre otros, generando una confianza entre el momento de enseñanza, sirviendo de apoyo y personalización de los conocimientos.

\section{Juegos educativos}

El juego desde sus inicios se ha caracterizado por proporcionar al individuo una sensación de libertad, autonomía y relajamiento durante su práctica, de igual forma Campos (2010), expresa que constituye una herramienta fundamental de aprendizaje que influye en todas las facetas de su personalidad y desarrollo; antropólogos, psicólogos y pedagogos, coinciden en afirmar que el juego junto a otras expresiones lúdicas se convierte en un elemento formativo de ser humano.

En referencia, el juego educativo es aquel juego que tiene implícita o explícitamente un objetivo educativo, es decir, una finalidad especifica en la que se adquiere un conocimiento y se retroalimenta un proceso. Para Silva (2015), este tipo de juegos 


\section{Ana Esther Oñate-Gonzalez}

puede ser empleado en diferentes niveles académicos, así como en diferentes rangos de edades. En este marco de ideas, el juego a nivel educativo ejerce una educación integral y un cumulo de experiencias que tiene un rol significativo en todo el proceso. Cabe aclarar que es más recomendable este tipo de juegos cuando existe un guía que de pautas para posteriormente llevar a cabio un aprendizaje significativo. Al respecto, Rodríguez (2006), considera que los juegos educativos tienen como finalidad el objetivo implícito o explicito para que los alumnos aprendan algo específico, un objetivo que explícitamente programa el docente con un fin de educación la persona que los diseña todo para que aprendan algo concreto de forma lúdica por ser un método de enseñanza de una forma estructurada para instruir o enseñar los contenidos escolares.

De la misma manera Gallardo (2018) indica que los juegos son actividades lúdicas, recreativas y placenteras que se practican a cualquier edad. Los niños juegan en sus primeros años de vida para divertirse, buscar afecto y crear solidaridad; y, al mismo tiempo, jugando desarrollan su fantasía, su imaginación y su creatividad y aprenden a vivir. Pero sus juegos no tienen aún normas específicas y surgen de manera espontánea, natural, sin aprendizaje previo. Más tarde comenzarán a practicar ya el juego reglamentado, es decir, dotado de una serie de normas que determinan no sólo las condiciones que se deben dar previas al inicio del juego, sino que regulan el desarrollo y terminación del mismo.

En este orden de ideas, los docentes de las instituciones educativas en Colombia deben ejercer los juegos educativos, con la finalidad de que los alumnos adquieran los conocimientos en algo específico, es decir es un método de enseñanza basándose en los contenidos escolares, incluyendo todas las facetas coincidiendo expresiones lúdicas en un elemento formativo de ser humano.

\section{Juegos libres}

En referencia a los juegos libres es una actividad se utiliza como diversión para su participación aprendiendo o adquiriendo diversos conocimientos. Para Rodríguez (2006), el juego libre es el hallazgo de nuevas cosas, de nuevas ideas que le permitirán al ser humano actuar de manera innovadora ante las situaciones 


\section{Ana Esther Oñate-Gonzalez}

encontradas. El juego libre es entonces una contraposición al juego dirigido; en este cada uno es capaz de establecer cómo, con quién, con qué y para qué quiere jugar, establece sus reglas y decide con que materiales y herramientas jugar. Este tipo de juego se puede ejecutar en cualquier contexto.

Por su parte, para Farías y Rojas (2010), dejar a los niños jugar con total libertad en el exterior es beneficioso para desarrollar su aprendizaje y sus habilidades sociales y emocionales, algo que les ayudará en el futuro. Las actividades al aire libre no sólo desarrollan mejores capacidades físicas, gracias a la práctica de deporte, sino que también implican la mejora de otras habilidades beneficiosas para los más pequeños. Ya de por sí, el ejercicio físico ayuda a que los niños ganen confianza en sí mismos, porque desarrollan sus capacidades y vencen sus miedos.

Además, indican que jugar en el exterior ayuda a los más pequeños a mejorar sus habilidades sociales, ya que están en contacto con otros niños y deben aprender a relacionarse, a compartir, a negociar, y en definitiva, a interactuar con los demás. En este sentido, jugar con otros niños también implica la necesidad de desarrollar las habilidades de liderazgo y cooperación, entre ellas aprender a dirigir, pero también a ceder, algo que será muy valioso para el futuro.

Asimismo, jugar al aire libre estimula la imaginación y la creatividad de los niños, ya que es un escenario idóneo para inventar juegos e imaginar situaciones. Las oportunidades de aprendizaje son numerosas en los espacios abiertos, donde los niños tienen la posibilidad y total libertad de observar, explorar y experimentar por sí mismos.

Al respecto, Jiménez (2009) señala que el juego libre se trata de una actividad natural del ser humano, en la que esta toma parte por la sola razón de divertirse y sentir placer, es decir, cuando comparten, cooperan y disfrutan al acompañamiento de los otros, se fortalecen en ellos los sentimientos de pertenencia al grupo social con el que comparten así como se va solidificando su sentimiento de solidaridad. De igual manera, David (2006) exponen en el juego ya sea individual o colectivo, el ser humano se afirma como tal se descubre y aprende una cantidad de acciones sociales que de alguna manera, le facilitaran actuar en la esfera cultural. En otras palabras, facilita las 


\section{Ana Esther Oñate-Gonzalez}

relaciones sociales, el intercambio de ideas y opiniones reconociendo las diferencias repetidas compartiendo entre otros aspectos.

Es por ello que, los docentes de las instituciones educativas deben propiciar los juegos libres con la finalidad de encontrar diversas ideas que permitirán actuar de manera innovadora ante las situaciones encontrada, es decir, el juego libre estableciendo las reglas y herramientas para jugar y aprender, compartir y disfrutar al acompañamiento de los demás teniendo pertinencia al grupo social.

\section{Juegos de Análisis}

En relación a los juegos de análisis Rodríguez (2006), hace referencia a las diferentes habilidades, destrezas y operaciones mentales que pone en práctica el individuo para conseguir un objetivo deseado. Este tipo de juego es poco frecuente en su utilización puesto que requiere de más concentración y dedicación para lograr un fin trazado. De igual manera, Jiménez (2009) señala que el juego de análisis es la manera más lúdica e interesante de estimular las funcionalidades del cerebro, haciendo referencias que las habilidades de mismo aumenten y así mejorar las capacidades intelectuales de un ser humano, en tal sentido ayudan ejercitar tu cerebro en las partes que están en falencias además de las cosas básicas.

Por otra parte, Suárez (2008) exponen que los juegos de análisis son basados en la realización de actividades en las cuales se demostraran las habilidades, operaciones mentales todo con la finalidad de conseguir el resultado deseado, de igual manera es necesario realizar la concentración y dedicación para el aumento de las capacidades intelectuales del estudiante fortaleciendo el ejercitar las partes del cerebro.

En relación a lo expresado, los docentes de las instituciones educativas de Colombia deben propiciar los juegos de análisis para una mejor enseñanza en cuanto los alumnos, para el aumento de las capacidades intelectuales, ejercitando el cerebro al realizar la concentración para la realización de las actividades a través del juego a pesar de su poca frecuencia en la utilización por parte de los docentes en el aula. 


\section{Ana Esther Oñate-Gonzalez}

\section{Juegos lógicos}

Al respecto, Rodríguez (2006) considera que los juegos lógicos son medios didácticos y de conocimiento que han sido diseñados por grandes pensadores, estructurados y sistematizados por educadores para contribuir al desarrollo de habilidades lógicas, razonamiento analítico y concentración a través de la estimulación y motivación de sus estudiantes para conseguir un proceso de enseñanza- aprendizaje de calidad. Este tipo de juego pone en práctica un sinnúmero de habilidades y operaciones mentales que le permitan encontrar la respuesta al problema planteado. Por lo general, se utilizan acertijos y juegos de bloque.

Por otra parte, Jiménez (2009) expresa que son los juegos que estimulan el desarrollo y la mente de los alumnos, algunos de ellos son los rompecabezas, como los laberintos o los juegos de las diferencias, así como los ejercicios intelectuales siendo útiles para los alumnos, suponiendo la forma de desarrollo reforzando las habilidades cognitivas y de aprendizaje, pero sobre todo divertida.

Asimismo, Suárez (2008) manifiesta que los juegos lógicos dan origen etimológico que le dan formas dos palabras al término del pensamiento lógico que se analizan en profunda en el pensamiento en base al significado de pensar. En tal sentido, los juegos lógicos es una herramienta agradable para los docentes de la educación ya que provee la formación de habilidades intuitivas de razonamiento y resolución de ejercicios lógicos brindando las ventajas de ser de fácil asimilación para el estudiante. Con relación a la definición de los juegos de pensamiento lógico Delval (2010) señaló que los juegos de pensamiento lógico, se constituyen en uno de los principales medios de aprendizaje, en la etapa de las operaciones concretas del desarrollo del niño, ya que, a través de ellos, estos, desarrollan gradualmente conceptos de relaciones causales, aprenden a discriminar, a establecer juicios, a analizar y sintetizar, e imaginar. Asimismo, para Morata (2011) el juego es una estrategia fundamental cuando se trata de atraer la atención de los niños. Cuando hablamos de trabajar con juegos, no nos referimos al solo hecho de jugar sin sentido, sino de utilizar el juego basado en un objetivo claro, la realización de competencias y desarrollo de capacidades.

También López y Garfella (2007) señalaron que muchos de los estudios e 


\section{Ana Esther Oñate-Gonzalez}

investigaciones actuales sobre la actividad lúdica en la formación de los procesos psíquicos convierten a los juegos de razonamiento lógico en una de las bases del desarrollo cognitivo del niño, ya que éste a través de los juegos, construye el conocimiento por sí mismo mediante la propia experiencia, experiencia que es esencialmente actividad, y ésta fundamentalmente juego. Los juegos de razonamiento lógico se convierten así en la situación ideal para aprender, y en la pieza clave del desarrollo intelectual.

Es por ello que en las instituciones educativas de Colombia los docentes deben emplear los juegos lógicos para el desarrollo de los alumnos a la contribución de las habilidades lógicas, el razonamiento analítico y la concentración estimulando al cerebro del mismo, aplicando los conocimientos diseñados para el proceso de enseñanza -aprendizaje, permitiendo o facilitando encontrar las respuestas esperadas.

\section{La Creatividad en el contexto educativo}

La creatividad se vincula con aspectos personales, sociales, culturales y económicos de los seres humanos. Está relacionada con la inteligencia, estilo cognitivo, contexto, innovación, solución de problemas, cambios del saber, desde la psicología a la economía, desde la ciencia y la tecnología admitiendo múltiples definiciones. Es en este contexto de transformaciones, la creatividad del docente no es vista como un don intrínseco sino como un aspecto a desarrollar en función del contexto social, de la gestión de centro, de la motivación docente, del trabajo colaborativo, entre otros. La misma es necesaria en las actividades, especialmente en las educativas, porque permite el desarrollo de aspectos cognoscitivos y afectivos importantes para el desempeño de docentes y discentes. Ante la posibilidad de tener en el recurso humano, el agente de cambio capaz de enfrentar los retos de una manera crítica, comprometida y diferente.

Por su parte, Rebollo (2010, p. 5), considera "un docente que estimula el desarrollo de la creatividad debe tener un pensamiento divergente utilizando el conocimiento previo de manera innovadora, generando el número de soluciones posibles ante una situación dada". Los profesores más creativos tienen ideas divergentes, se vean 


\section{Ana Esther Oñate-Gonzalez}

estimulados por su praxis pedagógica, apoyen a sus alumnos, permitiendo abordando temáticas a elección, aportando soluciones abiertas, promoviendo el diálogo y la discusión, incentivando la independencia entre los equipos de trabajo.

La autora describe ciertas habilidades directamente vinculadas al pensamiento creativo las cuales son:

1. Fluidez: Considerada como la facilidad para generar un gran número de ideas de calidad, de manera permanente y espontánea.

2. Sensibilidad: Denota la capacidad que poseen las personas creativas para descubrir diferencias o imperfecciones, dándose cuenta de lo que debe hacerse.

3. Originalidad: Se considera como la amplitud o disposición para producir de forma poco usual repuestas ingeniosas o novedosas, de gran interés y aporte a la comunidad.

4. Flexibilidad: Implica la capacidad de transformación, de cambio, de replanteamiento de una situación dada.

5. Elaboración: Es el nivel de detalle, desarrollo o complejidad de las ideas creativas.

Es la amplitud del sujeto para desarrollar, ampliar o embellecer las ideas.

6. Iniciativa: Es la capacidad humana para idear y emprender nuevas actividades para dirigir acciones, es la disposición personal para protagonizar, promover y desarrollar ideas.

Por su parte para Correa (2010, p. 2), la creatividad es definida como "la facultad de crear en general, o la capacidad para realizar obras artísticas u otras cosas por medio de la imaginación". En tal sentido, la creatividad es un fenómeno en el que intervienen elementos característicos de lo creativo como: espontaneidad, sentido del humor, curiosidad intelectual, iniciativa, intuición, independencia, inteligencia, persistencia, constancia y constituye un aspecto importante de la solución de problemas. A su vez, los individuos creativos son aquellos que dan lugar con frecuencia a productos creativos, es decir, originales, caracterizados por una combinación de inteligencia y personalidad en la que influyen las relaciones con otros profesionales, las circunstancias de trabajo y el apoyo de los que rodean al individuo creador e influyen significativamente en el proceso creativo.

En continuidad con la idea anterior, las mismas son creativas, valoran y buscan la 


\section{Ana Esther Oñate-Gonzalez}

originalidad, practican la autonomía, toleran la ambigüedad, entre otros. Para la creatividad parecen necesitarse actitudes que favorezcan la complejidad, así como un compromiso con el problema y una apertura a la exploración de enfoques innovadores. Un estilo cognitivo flexible para detectar problemas, donde se pueda conducir más el pensamiento creativo.

En tal sentido, De Bono (2009), relaciona a la creatividad con el pensamiento lateral siendo necesario ampliar la visión, enriqueciendo el uso de la información antes de pensar de manera vertical. La base de su método para desarrollar la creatividad consiste en introducir pautas como elementos activadores del pensamiento, produciendo un cambio de "penetración" y que proporcionan alternativas distintas y originales.

De igual manera el autor, sugiere el uso de herramientas del pensamiento, permitiendo, sacar a las personas de los extremos para explorar nuevos caminos, utilizar la información de otra manera y reestructurar sus patrones de los mismos. Igualmente, considera que la percepción es muy importante en las primeras etapas de todo proceso de pensamiento, favoreciendo la posibilidad de generar ideas que aun cuando parezcan ilógicas contribuyen a desarrollar la creatividad.

A la par, Churba (2007, p. 3), expresa que la creatividad "es la actitud y la aptitud para generar por un proceso creador nuevas ideas para descubrir nuevos significados, para inventar nuevos productos, nuevos servicios, para encontrar nuevas conexiones ya sea en el nivel individual o en el social". La creatividad es una cualidad necesaria para resolver muchos de los problemas que el alumno debe afrontar a lo largo de su vida. Según los autores Méndez y Sánchez (2012), es la capacidad de síntesis. Lograr reunir muchas cosas distintas en una sola. Las mejores ideas son las que nadie ha tenido antes. Las ideas, la creatividad, nacen de leer menos. Cuando lees, aprendes sobre las creaciones de los demás, pero no sobre las tuyas propias. Es necesario ser constante y divertirse, trabajar más que los demás. Así, todo el mundo puede crear, la clave está en las consecuencias de lo realizado, la creatividad se asocia a lo artístico, pero no se da solo ahí. Consiste en inventarse pequeñas formulas, ya sea para vender un producto o proyecto, a su vez, une diferentes conceptos de distintos campos. 


\section{Ana Esther Oñate-Gonzalez}

Al respecto, López (2009), la creatividad es "la capacidad de crear, de producir cosas nuevas, para llegar a conclusiones y resolver problemas en una forma original”. Así, la misma es el principio básico para el mejoramiento de la inteligencia personal y del progreso de la sociedad y es también, una de las estrategias fundamentales de la evolución natural. Es un proceso que se desarrolla en el tiempo y que se caracteriza por la originalidad, por la adaptabilidad y por sus posibilidades de realización concreta. Creatividad es la producción de una idea, un concepto, una creación o un descubrimiento que es nuevo, original, útil y que satisfacer tanto a su creador como a otros durante algún periodo.

Para el autor, Blanquez (2009, p. 3), es "Facultad de crear". A su vez, crear es "establecer, fundar, introducir por primera vez una cosa; hacerla nacer o darle vida, en sentido figurado. El concepto de creatividad engloba tal magnitud de rasgos, características, matices, entre otros, donde cada individuo tiene su propia concepción, sin errores, pero si diferente a la del otro grupo.

Por último, para la investigadora, el concepto de creatividad es diferente para diversos contextos donde, la actividad humana expresada en el concepto puede ser muy variada. En cualquier caso, pueden establecer límites para el empleo de esta palabra, aunque las delimitaciones son confusas, indeterminadas, relacionada con la innovación para generación de conceptos nuevos de diversos temas.

Ante lo expuesto, los docentes no desarrollan la creatividad de sus alumnos ni la de ellos, lo cual ha generado un sin número de obstáculos en el proceso de enseñanzaaprendizaje, dificultando la retroalimentación, pensamiento creativo, producción de ideas innovadoras, fluidez, originalidad, sensibilidad, flexibilidad y por último, la iniciativa que deben tener en el entorno escolar, sirviendo estos aspectos como elementos de interés al momento de triangular la información de la investigación que se desarrolla.

\section{Etapas del pensamiento creativo en los individuos}

El proceso creativo no es un proceso fácil pero no imposible, el proceso creativo es un proceso interactivo que conjuga un sinnúmero de habilidades. Es llegar a un principio siempre y en el cual se producen unos procesos intermedios que te permite 


\section{Ana Esther Oñate-Gonzalez}

llegar a una conclusión de que lo que se está desarrollando vale la pena. Se refiere a las fases que conllevan a la solución de un problema, las cuales están involucradas en un proceso creativo.

En tal sentido, la actividad de resolución de problemas señalando que tal actividad ligada al pensamiento creativo involucra una serie de etapas. De acuerdo con, Wallas (2014), en su libro "el arte del pensamiento" presentó uno de los primeros modelos de las etapas del pensamiento creativo. Consideraba que la creatividad es la que posibilita que las personas se adapten a los cambios del entorno y señalo las siguientes etapas del pensamiento, proponiendo las anteriores fases como un proceso y considera que tienen que cumplirse en un orden lógico.

Sin embargo, estas no son las únicas etapas que se mencionan acerca de la creatividad, puesto que Rodríguez (2008), propone seis (6) etapas del pensamiento creativo coincidiendo solo en dos de ellas con Wallas. Igualmente, el proceso creativo es muy complejo de explicar por ser un proceso no lineal, no concebido ni explicarse con exactitud una definición impresionable, formalizando de alguna manera el proceso creativo a su particular no es algo que este consensuado.

Asimismo, Whetten (2014), señala que las etapas de pensamiento creativo en todos los momentos de la vida se presenta situaciones y problemas los cuales se requieren ser solucionados y para que esto actuando el cerebro de la manera conjunta estableciendo un perfecto equilibrio entre los dos hemisferios, tanto lo lógico como el creativo. Asimismo, el desarrollo de la creatividad es muy importante para el día a día y trabajar con el conjunto del pensamiento creativo sus aspectos, caracterizas etapas entre otras.

Es por ello, que los docentes de las instituciones educativas de Colombia, deben emplear las etapas de pensamiento creativo al ejecutar la preparación, incubación, iluminación y verificación para la formalización de los procesos creativos a su particular en todos los momentos de la vida diaria en especial al momento de realizar las actividades escolares por ser la capacidad para el desarrollo de las nuevas ideas. 


\section{Ana Esther Oñate-Gonzalez}

\section{Preparación}

En referencia a la preparación es un período de inmersión consistente o no en un conjunto de cuestiones problemáticas que son interesantes y sucinta curiosidad. Asimismo, Whetten (2014), contiene recolección de fundamentos, ilustración del problema, generación de alternativas y análisis consistente de toda investigación aprovechable; la incompatibilidad entre la solución creativa y la solución analítica de problema es cómo se aborda la primera etapa. De hecho, es en este espacio que el entretenimiento en la solución creativa de problemas puede perfeccionar significativamente la validez debido a que las otras tres etapas no están paralizas a un compromiso mental consiente.

Según Valero (2006), habla del desarrollo como el pensamiento creador se inicia a causa de la entrada en escena de un problema. Es el choque con el problema, el considerar una especie de vacío o laguna. El rompimiento de un equilibrio debido a la presencia de las fuerzas antigénicas. Esta suele ser la etapa más prolongada ya que trata de ir almacenando investigación y documentación.

Por otra parte, García (2007), la elaboración para una creación plástica o visual reside en la compasión a las dificultades que conlleva el compromiso a realizar, la investigación de documentación gráfica o escrita o el rastreo de la propia presencia que seguramente dispondrá de imágenes y datos de interés; se delimita el problema en el cual se encamina la mente y examina sus extensiones.

También para Mihaly (1996) esta etapa consiste en la inmersión consciente o no, es un conjunto de aspectos problemáticos que generan curiosidad. Por esta razón, los docentes de las instituciones educativas de Colombia se deben propiciar la preparación para la disposición o arreglo de las cosas necesarias para realizar algo o para un fin determinado de la creatividad; Para emplear al proceso y al resultado de preparar para cumplir algo. Es decir, la preparación consiste en el proceso de recolección de información para definir el problema y la utilización de conocimientos adquiridos, así como la realización de los esquemas o estructuras. 


\section{Ana Esther Oñate-Gonzalez}

\section{Incubación}

La incubación es el período donde las ideas se agitan por debajo del umbral de la conciencia realizándose las conexiones inusitadas. De manera breve Valero (2006), narra de la incubación como el proceso que incluye en su mayoría la actividad mental inconsistente en que la mente combina los pensamientos no relacionados en la búsqueda de una solución. El esfuerzo consiente no participa. En esta fase se estudia toda la información recopilada el individuo se dedicará a otras actividades, pero su sub consiente estará trabajando en la posible solución. Whetten (2014), incluye en su mayoría la actividad mental inconsciente en la que la mente combina las inclinaciones no concernidos en la exploración de una tramitación. La voluntad consiente no advierte, se le conoce también con el nombre de periodo de frustración, porque el individuo busca soluciones al problema desde diferentes ángulos y a veces no encuentra la solución, frustrándose, durante esta etapa.

Por otra parte, García (2007), indica que es el momento en que se está gastando la creación. En la composición de una obra se hacen multitud de tanteos y pruebas con distintos materiales e instrumentos, se ordenan de diferentes formas los elementos sobre el soporte, se hacen hipótesis y se evalúan los posibles resultados. Asimismo, la incubación es interiorizada en el hemisferio derecho y parece que nada pasa externamente. En el mismo orden de ideas Mihaly (1996) indica que en esta etapa las ideas se agitan por debajo del lado consiente del individuo, y precisamente debido a esto surgen combinaciones inesperadas pues el lado lógico-consciente no opera en esta fase.

Por tal motivo en las Instituciones educativas de Colombia se debe propiciar la incubación ya que es esta fase solo se piensan las ideas aquí el problema es interiorizado en el hemisferio derecho y parece que nada pasa externamente. Es decir, la preparación consiste en la percepción de un problema y reunión de informaciones o cuestiones problemáticas que son interesante y se suscitan la creatividad.

\section{Iluminación}

En relación a la iluminación es considerada como la fase en la que circulan todas las ideas. Al respecto, García (2007), para el autor la iluminación o inspiración es en la 


\section{Ana Esther Oñate-Gonzalez}

que llega una solución al problema a lo planteado. Intuitivamente se opta por una entre las múltiples organizaciones plásticas posibles; la que se piensa que va a responder mejor a nuestro propósito expresivo, comunicativo o estético.

En tal sentido, Valero (2006), menciona de la iluminación que después de haber experimentado una especie de frustración viene la satisfacción de ver rápidamente, en un momento repentino e inesperado, la solución apetecida. Es en esta fase donde se prende el foco a la solución perfecta que influye en la mente. En tal sentido, busca inconsciente de la oscilación. De manera similar, Whetten (2014), afirma que la tercera etapa, iluminación ocurre o sucede cuando se reconoce o se articula una situación creativa. Cuando la idea creativa salta del procedimiento interior y se exterioriza al consiente. En esta fase se le consigue la solución al problema, generándose casualmente en ese momento ansiedades miedo, a quedarse en el vacío y a la incapacidad. Si también para Mihaly (1996) es cuando las piezas del rompecabezas encajan. En la vida real, pueden darse varias intuiciones mezcladas con períodos de incubación, es decir, las fases del proceso creativo no son lineales.

Por tal motivo en las Instituciones educativas de Colombia se debe favorecer la iluminación, puesto que en esta etapa se pone en marcha las ideas creativas pensadas solucionando problemas y va desde el subconsciente hasta el consiente a la hora de solucionar problemas. En tal sentido, se basa en la solución de irrumpir de golpe, cuando llega la luz a la oscuridad del proceso de incubación y las partes antes dispersas que se unen presentando un todo ordenado.

\section{Verificación}

Aunque se entiende que el acto creativo es un proceso unitario en el que estas fases se presentan sin interrupción y no siempre en el mismo orden procede, no obstante, hacer una descripción diferencial de las mismas. Por otra parte, García (2007), la verificación es aquella en la que contrastamos el resultado obtenido con el contenido de la institución; se comprueba que el plan o I idea eran realmente los correctos. De esta manera, para Valero (2006), la verificación es la idea luminosa impulsa a elaborar, a verificar y plasmar en algo concreto; el proceso señalado no es rígido pues a veces se interfiere las fases o remiten de nuevo de una a otra y no es raro ver como después 


\section{Ana Esther Oñate-Gonzalez}

de la iluminación se pasa de nuevo a la incubación para madurar determinados puntos aun oscuros.

Por otra parte, para Whetten (2014), la verificación es la etapa final de la creación, que incluye evaluar la solución relativa en relación con algún estándar de aceptación. Es la última etapa del proceso artista; la solución tiene que someterse a la detracción y la confirmación y así poder pulir. La denominación de esta novísima etapa ha sido contendida, ya que entiende relatar más a los procesos creativos de tipo probado; pero no así a los procesos primorosos. Es el proceso de valoración sobre el beneficio temporal del objeto o proceso de creación.

En palabras de Mihaly (1996) es cuando una persona sopesa si la intuición es valiosa y si vale la pena darle atención. Esta es la parte emocionalmente más difícil del proceso, cuando uno se siente más incierto e inseguro. Es cuando necesitamos la opinión de otros. Es cuando surgen preguntas como: ¿Es realmente original esta idea? ¿Qué pensarán mis colegas? Es el período de la autocrítica. El autor agrega otra etapa y es la evaluación la cual es la fase que lleva más tiempo y supone el trabajo más duro.

Por tal motivo en las instituciones educativas de Colombia se debe propiciar la verificación como ayuda de la creatividad a la solución propuesta, la gran idea tiene que ser comprobada verificada y para esto se investiga se busca la opinión de otras personas, de preferencia grupos afines, en donde se informa a la comunidad o a su medio ambiente para después recibir respuesta de aceptación, valoración, estima de las ideas producidas.

\section{METODOLOGÍA}

Para el presente estudio se utilizará un diseño de investigación cualitativa, la cual de acuerdo a Erlandson (2009), es el intento de un investigador de poner orden a un conjunto de fenómenos de tal forma que tenga sentido y pueda comunicar este sentido a los demás. Por ello, todas las decisiones a tomar durante el desarrollo del estudio pueden considerarse previamente, pueden planificarse y la concreción de ésta se realiza, por lo común, en un estudio de caso, el cual, de acuerdo a Denny (2008), se trata de un examen completo o intenso de una faceta, una cuestión o quizás los 


\section{Ana Esther Oñate-Gonzalez}

acontecimientos que tienen lugar en un marco geográfico a lo largo del tiempo.

De la misma manera Bernal (2006), refiere que en la investigación cualitativa no es prioritario medir, sino cualificar y describir el fenómeno social a partir de los rasgos determinantes, según sean percibidos por los elementos mismos que están dentro de la situación estudiada. Por su parte, en la definición de Martínez (2010), la investigación cualitativa trata de identificar, básicamente la naturaleza profunda de las realidades.

De aquí que lo cualitativo (que es el todo integrado) no se opone de ninguna forma a lo cuantitativo (que es solamente un aspecto) sino lo que implica integra, especialmente donde sea importante. El presente estudio se encuentra dentro de un enfoque de investigación cualitativo, seleccionado porque el conocimiento se basa en la fundamentación de las cosas sin afectar, ni pasar por desapercibida ninguna idea, por ello alcanza el conocimiento con precisión y certeza.

Asimismo, los diseños cualitativos se distinguen por orientarse a describir e interpretar los fenómenos y son adecuados para los investigadores que se interesan por el estudio de los significados de las acciones humanas desde la perspectiva de los propios agentes sociales. Se aborda el mundo personal de los sujetos (como interpretan las situaciones, qué significados tienen para ellos) no observables directamente ni susceptible de investigación experimental. En ese orden de ideas, Álvarez (2007) indica que el investigador estudia a las personas en el contexto de su pasado y de las situaciones en que se hallan.

Por consiguiente, la metodología que utiliza esta perspectiva de investigación se caracteriza esencialmente por un enfoque de diseño fenomenológico, por su carácter minimalista y flexible, es lo que supone partir de un plan de acción suficientemente flexible y abierta para acercarse al fenómeno, comunidad o situación a estudiar, atender a lo inesperado, recolectar la información necesaria.

\section{REFLEXIONES FINALES}

La lúdica y la creatividad son una necesidad y función del ser humano ya que posibilitan preparar y potenciar todos los procesos de desarrollo del ser humano; hacen posible los espacios abiertos a la incertidumbre, a partir de los conocimientos 


\section{Ana Esther Oñate-Gonzalez}

que sirven de base para nuevas creaciones; posibilitan nuevas miradas sobre la realidad y la construcción de alternativas cada vez más certeras.

La actividad lúdica ejerce una función de distensión, de esparcimiento, de diversión y libertad, permitiendo vivir en un micromundo relacionado con todo un universo simbólico lleno de significados sociales.

El juego debe ser un instrumento pedagógico indispensable en la actividad del maestro, pues liga el trabajo escolar al hacer libre, responsable y lúdico. El juego exige y posibilita el desarrollo del pensamiento creativo; los caminos no están dados de antemano, las reglas se conocen y se aceptan por acuerdo y, en el andar, nuevas reglas, nuevos juegos y nuevos caminos se construyen colectivamente.

La escuela colombiana puede dimensionar su trabajo cotidiano y, traspasando el estrecho límite de instancia reproductora de conocimientos y formadora de reproductores, ejercer acción transformadora, que se proyecte hacia la construcción de una sociedad justa. La escuela pensada por los maestros y por los niños y hecha realidad por ellos mismos, de conformidad con nuestras necesidades actuales.

\section{REFERENCIAS CONSULTADAS}

1. Álvarez, J. (2007). "Comunicación Interna, la Estrategia del Éxito". Razón y Palabra, 12, 56-59.

2. Andrade, Carruyo, Hernández, A (2012) El uso e importancia de las estrategias de aprendizaje en estudiantes de las Sedes Universitarias: Una Experiencia en Playa, Plaza y Cerro. $4^{\circ}$ Congreso Internacional Universidad'2004, La Habana, Cuba.

3. Beltrán, J. (2007). Estrategias de aprendizaje. En J. Beltrán y C. Genovard (Eds.),

4. Bernal, C. (2006). Metodología de la Investigación. México: Prentice Hall.

5. Blázquez O, A. (2009). La importancia de ser creativos. "Innovación y experiencias educativas". 17.

6. Campos J, (2010) Introducción a la Psicología del Aprendizaje. Perú: Editorial San Marcos. 
7. Churba, C. (2007). La Creatividad. Un Enfoque Dinamizador de las Personas y las Organizaciones. Buenos Aires: Dunken.

8. Correa, L (2010). Creatividad y Cerebro. Profit. España: Escenario.

9. Dansereau, D. F. (2009). Learning strategy research. En J.V. Segal, S.F. Chipman y Glaser (Eds.), Thinking and learning skills. Vol 1: Relating instruction to research. Hillsdale, NJ: Erlbaum.

10. David (2006). Desarrollo de videojuegos: Arquitectura de motor de videojuegos. Universidad de Castilla la Mancha.

11. De Bono, E (2009). Creatividad. 62 Ejercicios para Desarrollar la Mente. McQuaigGroup, Inc. España.

12. Delors J. (1997) La Educación encierra un Tesoro, Informe a la UNESCO de la Comisión Interamericana sobre la Educación para el siglo XXI México.

13. Delval, J. (2010). Las características del juego. España: Ediciones Ibérica.

14. Denny, T. (2008). Storytelling and educatinal understanding, address delibered ay Editorial Tirant Lo Blanch.

15. Erlandson, D. (2009). Doing naturalistic Inquity: a Guide to Methods.Newbury park.

16. Faria, B de Almeida., y Rojas, V. (2010). A inovação e o desinvestimento pedagógico na Educacão Física escolar: uma leitura a partir da teoría doreconhecimiento social. Motriz, 18(1), 120-129.

17. Gallardo, J. (2018) IV Teoría del Juego como recurso educativo. Congreso Virtual Internacional sobre innovación pedagógica y praxis educativa.

18. García, J. L. (2007) Los fundamentos de la educación social. Madrid: Magisterio Español.

19. Hidalgo R y Valverde H. (2009). Juguemos con cuentos y poesía. Costa Rica. Editorial Universidad Estatal a Distancia EUNED.

20. Jiménez, B. (2002) Lúdica y recreación. Colombia: Magisterio.

21. Jiménez, M. (2009). Aprendo a leer con alegría. En: Cuaderno Escenario № 2.

22. López, J. y Garfella M. (2007). El juego como recurso educativo. Valencia, España: 
23. López, R. (2009). Desarrollos conceptuales y operacionales acerca de la creatividad. Santiago de Chile: Universidad Central.

24. Martínez, M., (2010). "La capacidad creadora y sus implicaciones para la metodología de la investigación", Psicología (Caracas: UCV), vol.XII, núm.12,37-62.

25. Mendoza, H. (2015). La promoción de la lectura en inglés y su valoración por parte de los alumnos de noveno grado de la U.E. "Jacinto Regino Pachano" ubicada en Tocuyo de la Costa, Edo. Falcón”. [Trabajo especial de grado para obtener el 167 título de Magíster en Magister en Educación Mención Lectura y Escritura en la Universidad de Carabobo] Universidad de Carabobo. Valencia, Venezuela.

26. Mihaly, C (1996), "Creatividad el fluir y la psicología del descubrimiento y la invención", Barcelona, Paidós.

27. Morata, H. (2011). Psicología del aprendizaje matemático, Madrid, España: Editorial national meeting of International Reading Association. Houston, Texas. En Guba. E.G \& Lincoln. Y.S. (1981). Handbook of Qualitative Research. Thousands Oaks, CA. Sage Publications.

28. Rebollo, C (2010) La Creatividad Docente como Factor Generador de Nuevos Entornos de Aprendizaje en la Educación Media. Congreso Iberoamericano de Educación. Buenos Aires. República de Argentina.

29. Rodríguez, E. (2008). “Teatro y bienestar psicológico”. Mendoza. UDA.

30. Rodríguez, G. (2006). Motivación, estructuras de aprendizaje y rendimiento académico de estudiantes de ESO. Tesis doctoral. Universidad de A Coruña. Facultad de Ciencias de la Educación.

31. Silva, F. (2015). Introducción al psicodiagnóstico. Valencia: Promolibro.

32. Suárez, A, (2008). Errores de exactitud lectora. Trabajo presentado ante el Aula de Propuesta Educativa de la Universidad de Extremadura. España.

33. UNESCO (2010), Hacia las sociedades del conocimiento. Informe mundial, París, Organización de las Naciones Unidas para la Educación, la Ciencia y la Cultura, en: http://www.flacso.edu.mx/ colaboratorio/pdf/colaboratorio_unesco.pdf.

34.Valero, J (2006) Educación personalizada. Editorial Progreso, 1975 - 221 páginas. https://books.google.co.ve/books?id=skm8lfh6cOQC\&dq=Valero+,pensamient 
o+creador+se+inicia $+a+$ causa + de+la+entrada+en+escena\&source=gbs navli nks $\mathrm{s}$.

35. Wallas, G. (2014). The Art of Thought. New York: Harcart Brace.

36. Whetten, D (2014) Desarrollo de Habilidades Directivas. Disponible en: https://books.google.co.ve/books?id=PtcDi2ONvl8C\&dq=Whetten,++ pensamie nto+creativo+en+todos+los+momentos+de+la+vida+se+presenta+situaciones \&source $=$ gbs navlinks $s$.

\section{REFERENCES CONSULTED}

1. Álvarez, J. (2007). "Internal Communication, the Strategy of Success". Reason and Word, 12, 56-59.

2. Andrade, Carruyo, Hernández, A (2012) The use and importance of learning strategies in students of the University Venues: An Experience in Beach, Plaza and Hill. 4th International University'2004 Congress, Havana, Cuba.

3. Beltrán, J. (2007). Learning strategies. In J. Beltrán and C. Genovard (Eds.),

4. Bernal, C. (2006). Investigation methodology. Mexico: Prentice Hall.

5. Blázquez O, A. (2009). The importance of being creative. "Innovation and educational experiences". 17.

6. Campos J, (2010) Introduction to the Psychology of Learning. Peru: Editorial San Marcos.

7. Churba, C. (2007). The creativity. A Dynamic Approach of People and Organizations. Buenos Aires: Dunken.

8. Correa, L (2010). Creativity and Brain Profit Spain: Stage.

9. Dansereau, D. F. (2009). Learning strategy research. In J.V. Segal, S.F. Chipman and Glaser (Eds.), Thinking and learning skills. Vol 1: Relating instruction to research. Hillsdale, NJ: Erlbaum.

10. David (2006). Videogame development: Videogame engine architecture. Castilla-La Mancha university.

11. De Bono, E (2009). Creativity. 62 Exercises to Develop the Mind. McQuaigGroup, Inc. Spain. 


\section{Ana Esther Oñate-Gonzalez}

12. Delors J. (1997) Education contains a Treasury, Report to UNESCO of the InterAmerican Commission on Education for the 21st Century Mexico.

13. Delval, J. (2010). The characteristics of the game. Spain: Iberian editions.

14. Denny, T. (2008). Storytelling and educatinal understanding, address delibered and Editorial Tirant Lo Blanch.

15. Erlandson, D. (2009). Doing naturalistic Inquity: a Guide to Methods.Newbury park.

16. Faria, B de Almeida., And Rojas, V. (2010). A inovação e o pedagogical disinvestment in School Physical Education: a reading based on the theory of social recognition. Motriz, 18 (1), 120-129.

17. Gallardo, J. (2018) IV Game Theory as an educational resource. International Virtual Congress on pedagogical innovation and educational praxis.

18. García, J. L. (2007) The foundations of social education. Madrid: Spanish teaching.

19. Hidalgo R and Valverde H. (2009). Let's play with stories and poetry. Costa Rica. Editorial EUNED State University Distance.

20. Jiménez, B. (2002) Play and recreation. Colombia: Teaching.

21. Jiménez, M. (2009). I learn to read with joy. In: Notebook Scenario No. 2.

22. López, J. and Garfella M. (2007). The game as an educational resource. Valencia Spain:

23. López, R. (2009). Conceptual and operational developments about creativity. Santiago de Chile: Central University.

24. Martínez, M., (2010). "The creative capacity and its implications for research methodology", Psychology (Caracas: UCV), vol.XII, No. 1-27-7-62.

25. Mendoza, H. (2015). The promotion of reading in English and its assessment by the 9th grade students of the U.E. "Jacinto Regino Pachano" located in Tocuyo de la Costa, Edo. Falcon. " [Special degree work to obtain the 167 Master's degree in Magister in Education Mention Reading and Writing at the University of Carabobo] University of Carabobo. Valencia, Venezuela

26. Mihaly, C (1996), "Flowing creativity and the psychology of discovery and invention", Barcelona, Paidós. 


\section{Ana Esther Oñate-Gonzalez}

27. Morata, H. (2011). Psychology of mathematical learning, Madrid, Spain: Editorial national meeting of International Reading Association. Houston Texas. In Guba E.G \& Lincoln. Y.S. (nineteen eighty one). Handbook of Qualitative Research. Thousands Oaks, CA. Sage Publications.

28. Rebollo, C (2010) Teaching Creativity as a Generating Factor of New Learning Environments in Secondary Education. Iberoamerican Education Congress. Buenos Aires. Argentina republic.

29. Rodríguez, E. (2008). "Theater and psychological well-being". Mendoza UDA

30. Rodríguez, G. (2006). Motivation, learning structures and academic performance of ESO students. PhD thesis University of A Coruña. Faculty of Education Sciences.

31. Silva, F. (2015). Introduction to psychodiagnostic. Valencia: Promolibro.

32. Suárez, A, (2008). Reading accuracy errors. Paper presented to the Educational Proposal Classroom of the University of Extremadura. Spain.

33. UNESCO (2010), Towards knowledge societies. World report, Paris, United Nations Educational, Scientific and Cultural Organization, at: http://www.flacso.edu.mx/ colaboratorio / pdf / colaboratorio_unesco.pdf.

34. Valero, J (2006) Personalized education. Editorial Progreso, 1975 - 221 pages. Available https://books.google.co.ve/books?id=skm8lfh6cOQC\&dq=Valero+,thinking+cre ator+se+init+a+cause+de+la+entrada+en+escena\&source=gbs_navlinks_s.

35. Wallas, G. (2014). The Art of Thought. New York: Harcart Brace.

36. Whetten, D (2014) Development of Management Skills. Available at: https://books.google.co.ve/books?id=PtcDj2ONvl8C\&dq=Whetten,++ thinking + creative+en+all+++moments+de+la+life+se+presenta+situacione 